\section{INVITED PAPER:}

\section{Some thoughts about translational research}

\author{
THOMAS UNGER \\ CARIM - School for Cardiovascular Research, \\ Maastricht University, Maastricht, The Netherlands
}

DOI: $10.30824 / 2003-7$

"Translational research" is a buzzword of the day, but what does it mean? Let's approach the question in two different ways:

Educated in the traditional German Bildungssystem - the German word Bildung cannot be literally translated (there is that word again) into the English education; it goes beyond, sometimes defined as "what remains when you have forgotten everything that you have learned" - I would start with the etymological approach. The Latin verb transferre means that you carry something from one side to the other of, for instance, the Alps (cf. Gallia trans-alpina) or another obstacle. In doing this, there may be an advantage: you may conquer a new territory, you may learn something you have never heard of, you may combine your ancient, traditional knowledge with new insights and reach a higher level of wisdom. In order to achieve this, however, there must be something already in existence to be transferred, "a thing".

The second approach to defining "translation" is, of course, the one that most of us use: Wikipedia. What does the oracle say with respect to translational research?

Translational research (TR) - often used interchangeably with translational medicine - is a highly interdisciplinary field, the primary goal of which is to coalesce assets of various natures within the individual pillars in order to improve the global healthcare system significantly. The goal of translational medicine is to combine disciplines, resources, expertise, and techniques within these pillars to promote enhancements in prevention, diagnosis, and therapies. ${ }^{1}$

The citation refers to a 2014 article by Cohrs et al. "Translational Medicine definition by the European

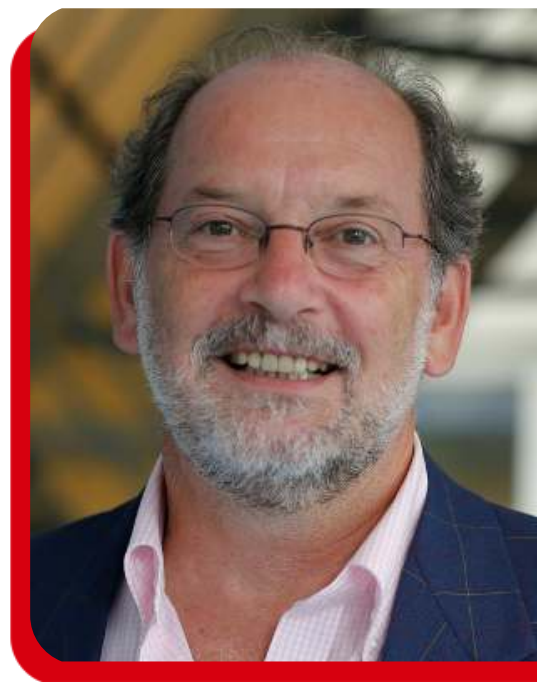

Society for Translational Medicine (EUSTIM)". EUSTIM further defines translational medicine as "an interdisciplinary branch of the biomedical field supported by three main pillars: benchside, bedside and community." 2

This gives us enough material to chew on for a few minutes.

Forget for a moment whether "translational research" is interchangeable with "translational medicine"; this is clearly not always the case, since both (bio)medical research and medicine are still distinct, although sometimes overlapping, areas. Let's concentrate on the interdisciplinarity of translational research. What this means is not only that scientists from different fields of basic research, e.g. a molecular biologist and a pharmacologist working together to develop a new therapeutic molecule, as this needs to be done anyway to be successful these days, but, rather, the interdisciplinary approach refers to the aforementioned pillars: first, from bench to bedside and then, maybe, to the community.

From bench to bedside: something, "a thing" that has been developed, an idea, a technique, a methodological approach, is transferred (here is the word again) to the clinic, is subsequently investigated in patients, and, if successful, further disseminated to the community.

The first step, from bench to bedside, certainly makes sense. Biomedical research draws its justification, to a large extent, from the principle of developing applications in order to help sick people or to prevent disease. We strive to invent; to develop new diagnostic, prognostic and therapeutic approaches; to drive the medical field forward and to benefit patients. 
The second step, translating the clinical application of a new drug or a new procedure or method to the community, is less straightforward. Indeed, current members of the "educated" community claim their right to receive all of the information available on biomedical progress, since, in the end, it is they, the taxpayers, who fund most of that research, at least to the extent that academia is involved. In this translational step, the sender and the receiver are often not well attuned to each other, which opens the door for misunderstandings and false expectations, since the community (receiver) cannot be expected to command the same knowledge and expertise as the information-providing scientists (sender), despite Wikipedia and the like. Good examples are the hope-raising monthly reports of novel treatments for Alzheimer's disease that seldom survive the drying of the ink on the paper on which they are printed (if printing is still used for such messages).

Therefore, while I agree that scientists need to open their Pandora's boxes to the scrutiny of the tax-paying community, I acknowledge that this pillar (here translational research has really turned into translational medicine) is quite a delicate issue, requiring serious efforts from both sides to synchronize sender and receiver.

\section{However, there is something elsein theworld of science, something which is entirely un- translational: the conduct of research driven solely by curiosity.}

Curiosity-driven research is not primarily applicationoriented. It springs from the zest to discover what is holding the world together, in a first approach, without respecting boundaries or considering consequences. In my view, scientists need to be given the chance to follow their curiosity, sometimes even serendipity, to think the hitherto unthinkable, to bind together thoughts and approaches previously separated, to have never-heard-of, fantastic ideas under the morning shower or while shaving, and try them out.

When the great scientific discoveries and achievements of the past are evaluated, many, if not all, were born out of a combination of the knowledge of the time with one or more unconventional ideas, resulting in the creation of a new paradigm, a new "thing".

One example, in which I participated myself, may serve to illustrate this: the venom of the Brazilian snake Bothrops jararaca contains certain peptides. Brazilian researchers found out that one or more of these peptides inhibit, among other things, the enzymatic degradation of another peptide present in our body, bradykinin, thus potentiating the actions of the latter. This was an interesting finding, but in and of itself, not sensational. The "living spirit" entered the game when somebody thought of linking the bradykinin degradation to another well-known peptide system, the renin-angiotensin system (RAS), by demonstrating that the bradykinin degrading enzyme, kininase II, was identical to the angiotensin converting enzyme (ACE), which splits off the blood pressure increasing peptide, angiotensin II, from its precursor. With this intellectual bridge to the RAS, the ground was paved for the development of non-peptide small molecules, ACE-inhibitors, which inhibit the formation of angiotensin II (and potentiate kinins). ACE inhibitors were subsequently introduced into the clinic with tremendous success for all kinds of cardiovascular indications and, even, beyond. What is important in this story, however, is this: years of research passed within a curiosity-driven period without translational moves, until the time and the "thing" were ready for drug development, i.e. the translational, application-oriented phase.

The above example, and many others, tells us that there seems to be a pre-phase driven by curiosity and creativity until the product of this phase finally enters the translational path. Is this curiosity-driven first step a prerequisite for translation? I would say, in most cases, yes: you need to create the "thing", a (drug)target or a novel method or procedure, first and then shape it into an object of translation and render it clinically applicable. On the other hand, does the product of curiosity driven research always end up in translation? Not necessarily, but it happens quite often. To sharpen the argument: does curiosity-driven research inevitably have to end in translation because researchers have a responsibility towards official funding sources or society at large? My answer to this is a definite no.

Curiosity-driven research needs to exist in its own right, not merely because it's the predecessor of the translational. It exists as an expression of the irrepressible human drive to deepen our knowledge about the world, as an expression of the living human spirit. It may even possess an intrinsic aesthetic value. Similarly, the results of our scientific curiosity have their own value, whether or not they will, in the short or long term, engender translational exercises or even give rise to ethical concerns with which scientists and the community will have to deal. 
These results serve what is called "gain of knowledge" and are regarded as inherently valuable in our culture. Perhaps not coincidentally, the landmark publications in the leading scientific journals, such as Nature, Science, Cell and the like, usually deal with topics in the "curiosity driven" category.
Thus, the call is out: copiously water all of the flowers growing in the field of creative cardiovascular research; watch them carefully, but let them bloom in their own right; don't be disappointed if not all of them can be picked and bound together, but, simultaneously, help those selected on their way to become part of the rich bouquet of translational medicine.

Thomas Unger - thomas.unger@maastrichtuniversity.nl

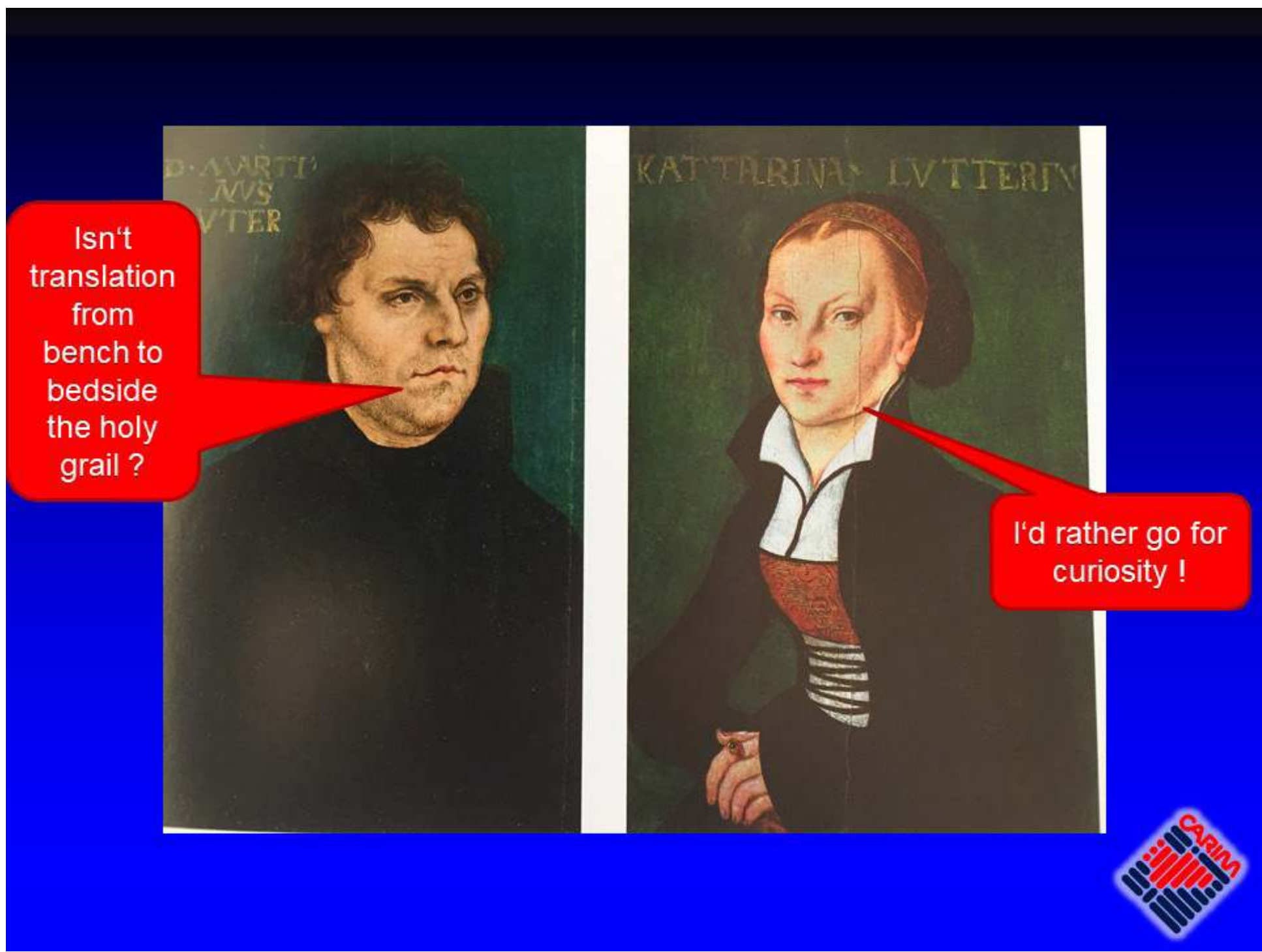

'Source: https://en.wikipedia.org/wiki/Translational_research

${ }^{2}$ Cohrs, Randall J.; Martin, Tyler; Ghahramani, Parviz; Bidaut, Luc; Higgins, Paul J.; Shahzad, Aamir. "Translational Medicine definition by the European Society for Translational Medicine". New Horizons in Translational Medicine. 2 (3): 86-88. doi:10.1016/j.nhtm.2014.12.002 